\title{
Early humps in WZ Sge stars
}

\author{
Y. Osaki ${ }^{1}$ and F. Meyer ${ }^{2}$ \\ 1 Faculty of Education, Nagasaki University, Nagasaki 852-8521, Japan \\ 2 Max-Planck-Institut für Astrophysik, Karl Schwarzschild Str. 1, 85740 Garching, Germany
}

Received 7 November 2001 / Accepted 6 December 2001

\begin{abstract}
Photometric humps in outburst that are locked with the binary orbital period have been observed exclusively in the early phase of outbursts of WZ Sge stars. It is suggested that this "early hump" phenomenon is the manifestation of the tidal 2:1 resonance in accretion disks of binary systems with extremely low mass ratios. The "early humps" can be understood by the two-armed spiral pattern of tidal dissipation generated by the 2:1 resonance, first discussed by Lin \& Papaloizou (1979). The tidal removal of angular momentum from the disk during outbursts of dwarf novae, an important feature, is discussed in the context of the disk instability model. The ordering of tidal truncation radius, the 3:1 and 2:1 resonance radius in systems of different mass ratio naturally leads to a classification of dwarf nova systems in three groups according to their mass ratio. The WZ Sge stars are those systems which have the lowest mass ratios and are therefore characterized by "early humps".
\end{abstract}

Key words. accretion disks - cataclysmic variables - stars: dwarf novae - stars individual: WZ Sge

\section{Introduction}

Several different types of periodic photometric humps are observed in light curves of dwarf novae both in outbursts and in quiescence. The most well known among them are the "orbital humps" in quiescence, which are repeated with the binary orbital period, and the "superhumps" observed during the superoutburst of SU UMa-type dwarf novae, which are repeated with a period slightly longer than the binary orbital period. Both types of humps are now well understood; the orbital hump is due to the phasedependent visibility of the hot spot located at that place of the accretion disk where the in-falling stream from the secondary star collides with the disk rim while the superhump is due to intrinsic light variation produced by tidal dissipation on an eccentric precessing disk in SU UMa stars (see e.g. Warner 1995).

Different from these is another type of photometric humps recently recognized as "early humps" observed in the first about ten days of the outbursts in WZ Sge stars. These humps repeat with the binary orbital period but they only appear in the early phase of outburst in WZ Sgetype dwarf novae exclusively and they are replaced by the ordinary "superhumps" in the later phase of the outburst. The "early hump" phenomenon was first discovered in the 1978 outburst of WZ Sge itself by Patterson et al. (1981) and has been observed consequently in other WZ Sge systems: the 1995 outburst of AL Com

Send offprint requests to: Y. Osaki,

e-mail: osaki@net.nagasaki-u.ac.jp
(Kato et al. 1996; Patterson et al. 1996); the 1992 outburst of HV Vir (Leibowitz et al. 1994) and the 1996 outburst of EG Cnc (Matsumoto 1998). These "early humps" have either been called "outburst orbital humps" by Patterson et al. (1996) or "early superhumps" by Kato et al. (1996). The different designation reflects a different interpretation of the phenomenon: Patterson et al. $(1981,1996)$ favor a "super-hot spot" interpretation in which early humps are due to a brightened hot spot which in turn is due to an enhanced mass transfer from the secondary star during outburst (Patterson et al. 1981), while Kato et al. (1996) favor an "early superhump" interpretation in which early humps are a premature form of the true superhumps. Here we call these humps simply "early humps" to avoid any particular interpretation.

In July 2001 WZ Sge (the proto-type of its group) underwent an unexpected full-scale outburst, 10 years earlier than expected with its former 33 years recurrence period. The star was caught on the rising branch of its outburst light curve. It was extensively observed by professional and amateur astronomers. Beautiful light curves showing "early humps" with an initial amplitude reaching $0.5 \mathrm{mag}$ and then decreasing a few tenths were observed (VSNET 2001: http://www.kyoto-u.ac.jp/vsnet/DNe/ wzsge01.html).

In this investigation we first suggest that the early humps exclusively observed in the early phase of outbursts in WZ Sge stars are most likely a manifestation of the 2:1 resonance in the accretion disk in these systems with extremely low mass ratios (Sect. 2). The amplitude of the 
early humps in the light curve is discussed in Sect. 3. In Sect. 4 we then present a general discussion of angular momentum removal from the accretion disk during outbursts of dwarf novae based on the disk instability model. This leads (Sect. 5) to a new subdivision in the dwarf novae unification model of Osaki (1996) which allows to distinguish between ordinary SU UMa stars and WZ Sge stars as those with the lowest mass ratios exhibiting "early humps" and "echo outbursts".

\section{Early humps: A manifestation of the tidal 2:1 resonance}

One of peculiarities of WZ Sge stars is the rather late appearance of the superhumps. In the 1987 outburst of WZ Sge, the regular superhumps first appeared ten days after the start of the outburst, while in ordinary SU UMa stars they usually develop within only a few days. The late development of superhumps in WZ Sge stars can be understood by the low growth rate of the eccentric tidal instability in these systems. Lubow (1991) showed analytically that the eccentricity growth rate is proportional to the square of the binary mass ratio $q$, if other conditions are kept the same. This very slow growth in the case of extremely low mass ratio systems is also confirmed by numerical simulations (Hirose \& Osaki 1990; Whitehurst 1994).

If in WZ Sge stars the eccentric disk is not yet well developed in the early phase when the major outburst has already started, the disk must expand well beyond the $3: 1$ resonance radius because nothing removes the angular momentum (released by the accreting matter). As demonstrated below, in binary systems with the extremely low mass ratio of WZ Sge stars, the disk would expand well beyond another resonance radius, the 2:1 resonance radius. In such a case the $2: 1$ resonance acts to truncate the disk. The 2:1 resonance is a very strong resonance as the periodic tidal force acting upon the disk resonates with the two-armed $(m=2)$ wave pattern in the Keplerian disk, i.e., the inner Lindblad resonance.

In fact, more than twenty years ago, Lin \& Papaloizou (1979) showed that the accretion disk in binary systems with extremely low mass ratio is truncated at the $2: 1$ resonance radius and that near the resonance, a strong twoarmed dissipation pattern forms. We here suggest that the tidal effect due to the 2:1 resonance is responsible for the "early humps" observed in WZ Sge stars.

The two-armed dissipation pattern produced by the 2:1 resonance was shown in Fig. 3a in Lin \& Papaloizou (1979). From this figure we can find that the strongest dissipation appears around 0.7 in the binary orbital phase while the second peak appears around 0.2 where phase zero is defined at the conjuction of the secondary star in front of the primary white-dwarf, i.e., the eclipse center. This is in good agreement with the observed phases of the double humps in the $2001 \mathrm{WZ}$ Sge outburst. On the other hand, in the super-hot spot model proposed by Patterson et al. (1981), the spot in outburst would have to be displaced by 60 degrees to the downstream direction from its position at quiescence, a rather unlikely possibility. The double-hump nature of the early humps also is difficult to explain by the hot spot model. The interpretation of early humps as a premature form of superhumps is clearly ruled out because early humps are repeated with the binary orbital period and not with the ordinary superhump period which is always longer than the orbital period by about one percent (or a few percent in the case of ordinary SU UMa stars) and because the amplitudes of the early humps were found to be larger than that of the ordinary superhumps in the 2001 WZ Sge outburst. Furthermore, as to the two other interpretations of the early humps, i.e., the super-hot spot model and the early superhump model, it has remained to be demonstrated why the early humps appear exclusively in those systems with extreme mass ratios.

\section{The amplitude of the early humps in the light curve}

In the 2001 outburst of WZ Sge, large-amplitude periodic humps were observed near the maximum with an initial amplitude of $0.5 \mathrm{mag}$ but then settling to an amplitude of a few tenths of a magnitude. Basically the same phenomenon was observed in the 1995 outburst of AL Com but with a much lower amplitude. We interpret this phenomenon as a two-step process, an initial transient adjustment stage of the disk and a more or less quasi-steady state in the later stage.

We first discuss the initial transient stage. Let us consider what would happen if the quiescent disk suddenly turns to a fully viscous state. In our picture of WZ Sge stars, the viscosity in the quiescent disk is extremely low. In the extreme case, material transfered from the secondary star is accumulated simply in a torus with its radius given by the circularization radius (Lubow-Shu radius). The circularization radius is given by

$R_{\mathrm{cir}} / a=(1+q)\left(R_{\mathrm{L}_{1}} / a\right)^{4}$

where $a, q=M_{2} / M_{1}, R_{\mathrm{L}_{1}}$ are the binary separation, the binary mass ratio, and the distance from the primary white dwarf to the inner Lagrangian point, respectively. The distance to the Lagrangian point, $R_{\mathrm{L}_{1}}$, is given by (see, e.g., Warner 1995)

$R_{\mathrm{L}_{1}} / a=1-w+\frac{1}{3} w^{2}+\frac{1}{9} w^{3}$

where

$w^{3}=\frac{q}{3(1+q)} \quad$ for $\quad q \leq 0.1$.

When an outburst occurs, the torus spreads out into the disk. Under the assumption of angular momentum conservation the outer edge of the resultant disk will then be given by $R_{\text {outer edge }} / a=(7 / 5)^{2} R_{\text {cir }} / a$. Here we have assumed the surface density profile $\Sigma=r^{-3 / 4}$ of a steady hot disk with free-free opacity whose mean specific angular 


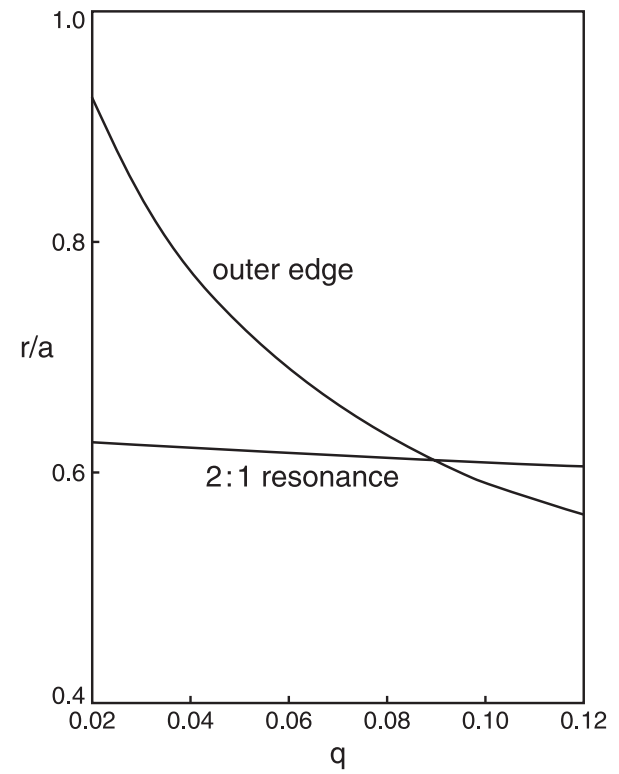

Fig. 1. Outer edge of the disk and radius of the 2:1 resonance, measured in units of binary separation $a$, as a function of the mass ratio $q$.

momentum, $j_{\mathrm{m}}$, is given by $j_{\mathrm{m}}=(5 / 7) \sqrt{G M_{1} R_{\text {outer edge }}}$. This is to be compared with the $2: 1$ resonance radius given by $R_{2: 1} / a=\left(2^{2}(1+q)\right)^{-1 / 3}$.

Figure 1 illustrates the outer edge of the disk and the $2: 1$ resonance radius as a function of mass ratio. We find that the outer edge of the disk exceeds the $2: 1$ resonance radius in the low mass-ratio systems with $q$ less than 0.08 . In those systems, the 2:1 resonance ensues, resulting in the two-armed spiral shocks and strong tidal torques acting on the disk. The extra angular momentum of the disk will be rapidly removed from the disk. We find from Fig. 1 that the extra angular momentum to be gotten rid off becomes greater as the binary mass ratio becomes smaller. The tidal dissipation luminosity resulting from the transfer of angular momentum by the $2: 1$ resonance is given by

$$
L_{\mathrm{tid}}=\left(\Omega_{\mathrm{K}}-\Omega_{\mathrm{orb}}\right) \dot{I}
$$

with $\Omega_{\mathrm{K}}=\left(G M_{1} / r_{\mathrm{d}}^{3}\right)^{1 / 2}$ Kepler angular frequency, $\Omega_{\mathrm{orb}}=$ $\left(G M / a^{3}\right)^{1 / 2}$ orbital angular frequency, $M=(1+q) M_{1}$ total mass and $r_{\mathrm{d}}$ the outer disk radius, $\dot{I}$ rate of angular momentum removed tidally from the accretion disk. At the earliest phase of the outburst, the rate of tidal removal of angular momentum from the disk, $\dot{I}$, must be much larger than that of the later phase discussed below because of the extra angular momentum of the disk to be removed.

After this intial transient phase is ended, a slower removal of angular momentum by the $2: 1$ resonance will follow. Let us estimate the amplitude of the early hump in this second stage expected from this 2:1 resonance model.

For this we compare the 2:1 resonance luminosity (i.e. the non-axisymmetric component of the dissipation) with the luminosity of the accretion disk (i.e. the axisymmetric component of the dissipation)

$L_{\mathrm{d}}=\frac{1}{2} \frac{G M_{1} \dot{M}}{r_{\mathrm{WD}}}$

where $r_{\mathrm{WD}}$ is the radius of the central white dwarf. In steady state the rate $\dot{I}$ of angular momentum transfer is equal to the rate of angular momentum released in the disk,

$\dot{I}=\dot{M} \sqrt{G M_{1} r_{\mathrm{m}}}$.

Here $r_{\mathrm{m}}$ is that radius where the Kepler specific angular momentum is equal to mean specific angular momentum of the disk. For the surface density profile $\Sigma \sim r^{-3 / 4}$ as before one obtains $r_{\mathrm{m}}=\left(\frac{5}{7}\right)^{2} r_{\mathrm{d}}$. At the $2: 1$ resonance $\Omega_{\text {orb }}$ is $\Omega_{\mathrm{K}} / 2$. Inserting these values into Eq. (4) we obtain

$L_{\mathrm{res}}=\frac{5}{14} \frac{G M_{1} \dot{M}}{r_{\mathrm{d}}}$.

We then find that the ratio of the $2: 1$ resonance luminosity to the disk luminosity is

$\frac{L_{\mathrm{res}}}{L_{\mathrm{d}}}=\frac{5}{7} \frac{r_{\mathrm{WD}}}{r_{\mathrm{d}}} \simeq 0.03$

where we took $r_{\mathrm{WD}}=10^{9} \mathrm{~cm}, r_{\mathrm{d}}=10^{10.4} \mathrm{~cm}$. This value is already of the right order of magnitude to explain the amplitude of the early humps observed in other WZ Sge systems, e.g. AL Com: 0.05 mag, HV Vir: 0.1 mag, EC Cnc: $0.02 \mathrm{mag}$, provided that the bolometric corrections for the disk luminosity and for the resonance luminosity are similar.

The following consideration estimates the relations in more detail. By integrating the bolometrically corrected (Allen 1973) contributions of individual rings of a standard accretion disk and comparing the resulting visual luminosity with the bolometric one one can derive a bolometric correction for the disk as a whole.

For the parameters as above and an estimated accretion rate of $\dot{M}=10^{-7} M_{\odot} /$ yr for the early outburst phase of WZ Sge we obtain

$\frac{\left(L_{\mathrm{d}}\right)_{\mathrm{V}}}{L_{\mathrm{d}}}=10^{-0.4(\mathrm{BC})_{\mathrm{d}}}$

with $(\mathrm{BC})_{\mathrm{d}} \simeq 3.8$ where the subscript $\mathrm{V}$ denotes the visual luminosity and $\mathrm{BC}$ is the bolometric correction.

For the 2:1 resonance luminosity the bolometric correction is determined by the effective temperature which is obtained from the luminosity and the area from which it is radiated. For the latter we estimate a total azimuthal extent of $\pi r$ (see Lin \& Papaloizou 1979) and assume a width of $4 H$ where $H$ is the scaleheight for the obtained temperature. With the parameters of WZ Sge as above this gives $\left(T_{\text {eff }}\right)_{\text {res }} \simeq 10^{4.2} \mathrm{~K}$ and a bolometric correction $(\mathrm{BC})_{\mathrm{res}} \simeq 2.6$.

The ratio of the visual luminosity from the resonance dissipation to that from the disk is then, larger 
than that of the bolometric luminosities by the factor $10^{-0.4\left[(\mathrm{BC})_{\text {res }}-(\mathrm{BC})_{\mathrm{d}}\right]} \simeq 3$. With Eq. (8) we obtain

$$
\frac{\left(L_{\mathrm{res}}\right)_{\mathrm{V}}}{\left(L_{\mathrm{d}}\right)_{\mathrm{V}}}=\simeq 0.09 \text {. }
$$

In addition a geometrical effect has to be considered. In order to determine the hump amplitude we have to know the orientation of the radiating surfaces with respect to the direction towards the observer. We assume that half of the spiral dissipation pattern surface faces upwards and downwards and half points radially. We account for a slightly uneven distribution of the two armed spiral dissipation pattern by attributing $2 / 3$ of the tidal dissipation to the major arm and $1 / 3$ to the minor arm. Maximum light will be seen at the orbital phase when the major arm is facing in our direction, minimum light about a quarter of the period later when the surfaces pointing radially are invisible. This gives a relative amplitude of the luminosity variation

$$
\frac{\frac{2}{3}\left(L_{\mathrm{res}}\right)_{\mathrm{V}} \cdot \sin i}{\left[\left(L_{\mathrm{d}}\right)_{\mathrm{V}}+\frac{1}{2}\left(L_{\mathrm{res}}\right)_{\mathrm{V}}\right] \cos i}=0.05 \tan i
$$

where $i$ is the inclination angle of the disk, $\pi / 2-i$ that of the radially pointing surface at maximum. The expected hump amplitude then becomes

$\Delta m_{\mathrm{V}}=2.5 \log (1+0.05 \tan i)$

and for an inclination angle for WZ Sge of $i=75^{\circ}$ (Smak 1993)

$\Delta m_{\mathrm{V}} \simeq 0.2$,

is in good agreement with the hump amplitude observed for WZ Sge of $\left(\Delta m_{\mathrm{V}}\right)_{\mathrm{WZ}}=0.2$ to 0.15 after the first two days.

WZ Sge is the only eclipsing system among the known WZ Sge stars. The other non-eclipsing systems must have lower inclination, leading to lower values for the hump amplitude, e.g. $\Delta m_{\mathrm{V}}=0.1$ for $i=60^{\circ}$ and $\Delta m_{\mathrm{V}}=0.03$ for $i=30^{\circ}$. This fits well to the observed range of $\Delta m_{\mathrm{V}}$ in these other systems mentioned above.

\section{Angular momentum removal from the disk in dwarf novae}

The cyclic behavior of outburst/quiescence in dwarf novae is most naturally understood by the disk instability model (Osaki 1974). In this model, mass supplied from the secondary star to the disk is not accreted onto the central white dwarf but rather is mostly stored within the disk during quiescence. The material accumulated in the disk is then suddenly accreted onto the central white dwarf during outburst due to an intrinsic instability within the disk. The intrinsic instability has been identified as the thermal instability in the disk, which produces a jump in disk viscosity (Meyer \& Meyer-Hofmeister 1981; for the general review of theoretical aspects of the disk instability model, see, e.g., Cannizzo 1993 and Osaki 1996).
Thus the cyclic behavior of dwarf novae is well understood as a process of interchanging mass accumulation and drainage in the disk. Equally important here is the cyclic variation of the total angular momentum in the disk. Angular momentum also accumulates during quiescence of dwarf novae. When an outburst occurs, the increase of viscosity leads to accretion onto the central white dwarf. However, in order to conserve angular momentum some material must move outward to radii of higher Kepler angular momentum giving rise to an expansion of the disk. The angular momentum brought outward will finally be returned to the orbit of the binary by tidal torques from the secondary star acting in the disk. It is thus understood that in a time-average over the outburst cycle a steady state is established for the total angular momentum of the disk in a same way as for the total mass of the disk.

In ordinary U Gem-type dwarf novae, a quasi-periodic outburst/quiescence cycle repeats in which the disk expands to the tidal truncation radius during an outburst. There extra angular momentum can be returned to the binary orbital motion via tidal torques. The accretion disk is then truncated at this radius. During quiescence, the disk radius gradually decreases since the matter added to the disk from the secondary star has a specific angular momentum lower than that at the disk edge. This gives rise to a cyclic variation of the disk radius. This picture fits very well to the observed variation of the disk radius in dwarf novae (Anderson 1988; Ichikawa \& Osaki 1992).

Paczynski (1977) calculated the tidal truncation radius as that of the last non-intersecting orbit of a test particle around the central star in the binary potential. His results agree fairly well with an analytic treatment of the tidal torques in the binary by Papaloizou \& Pringle (1977) who showed that tidal torques increase strongly with an increase of the radial coordinate in the disk.

For the angular momentum removal from the disk a new aspect emerged with the discovery of the tidal instability by Whitehurst (1988). In this instability, an accretion disk is deformed to an eccentric elliptic shape and then precesses progradely in the inertial coordinate system. When the eccentric disk is developed a very efficient removal of angular momentum from the disk is supposed to occur. The 3:1 resonance in the accretion disk, responsible for the tidal instability, is only possible in binary systems with a ratio $q=M_{2} / M_{1}$ of the secondary to the primary mass less than about 0.25 . This is because only in these low mass-ratio systems the tidal truncation radius is large enough to accommodate the $3: 1$ resonance radius within its boundary.

By combining the tidal instability with the thermal instability, Osaki (1989) proposed the thermal-tidal instability model (called TTI model) for the superoutburst cycle of SU UMa-type dwarf novae. In this model, the short normal outbursts occur as long as the disk radius is small and the mass accreted during the normal outbursts is less than that accumulated during quiescent intervals. Mass and angular momentum of the disk gradually build up. 
Thus during the sequence of normal outbursts the disk radius gradually increases until a final normal outburst drives the outer edge of the disk beyond the 3:1 resonance radius, triggering the tidal instability. Enhanced tidal removal of angular momentum in the eccentric precessing disk then keeps the disk in a hot state longer than a normal outburst does. This explains the long duration of the superoutburst and the superhump phenomenon. The typical supercycle length of SU UMa stars is a few hundred days, i.e., less than about a year.

The WZ Sge stars are an extreme case of SU UMa stars with long supercycles lasting decades. Besides a long recurrence time, WZ Sge stars exhibit several other unique characteristics, which are discussed by various workers (for modeling see Osaki 1995). It is thought that these systems have mass transfer rates lower than the ordinary SU UMa stars, $10^{15} \mathrm{~g} / \mathrm{s}$ for WZ Sge stars versus $10^{16} \mathrm{~g} / \mathrm{s}$ for the ordinary SU UMa stars. During quiescence the disk viscosity is very low (Smak 1993) which can be understood as to be related to the fact that the secondary stars are brown dwarfs without magnetic activity (Meyer \& MeyerHofmeister 1999), mass ratios $q=M_{2} / M_{1}$ less than 0.1, most likely as low as 0.03 . The "echo outbursts", a repetitive rebrightening after the main outburst, beautifully established during the 2001 outburst, are also related to the low viscosity (Osaki et al. 2001).

Recently Hellier (2001) made an interesting suggestion that some of peculiarities of WZ Sge stars and some of ER UMa stars can be understood within the TTI model by considering a possibility of rather weak tidal torques in the eccentric disk, in those with extreme mass ratios. He suggested that tidal dissipation in these systems is too weak to sustain the disk in the hot state long enough. A premature shut-down of the superoutburst could be responsible for "echo outburst" observed after the end of the main outburst in EG Cnc. Indeed Osaki et al. (2001) showed that the viscosity decrease related to the disk cooling after the main outburst and magnetic field decay causes the repetitive rebrightening of EG Cnc.

As discussed in Sect. 3, in binary systems like WZ Sge stars with the extremely low mass ratio, of $q \leq 0.08$, once an outburst occurs, the outer edge of the hot viscous disk exceeds the 2:1 resonance radius, thus exciting the strong two-armed dissipation pattern due to the 2:1 resonance, which now can remove angular momentum from the disk. The WZ Sge stars are thus those systems which are characterized by "early humps". Since the 3:1 resonance radius is smaller the 2:1 resonance radius, the tidal eccentric instability due to the $3: 1$ resonance still operates in those systems even though its growth rate is rather low. Eventually (after about ten days from the outburst maximum) the precessing eccentric pattern characterized by "ordinary superhumps" has grown to a sufficient amplitude. "Early humps" are now replaced by "ordinary superhumps" because the disk's outer edge does not need to reach the 2:1 resonance radius any more as the tidal eccentric pattern due to the 3:1 resonance can now remove angular momentum from the disk. Our interpretation is

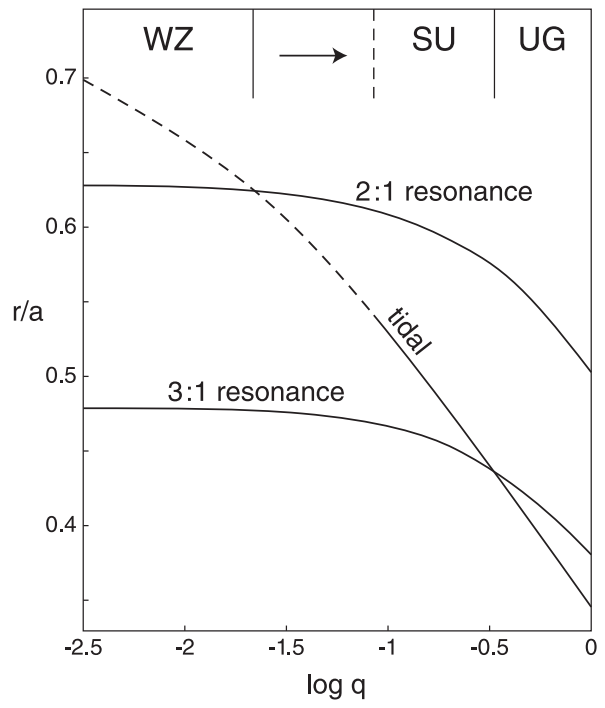

Fig. 2. Tidal truncation radius together with the radii of the $2: 1$ and $3: 1$ resonance, measured in units of binary separation $a$, as a function of the mass ratio $q$ and dividing lines between the three classes of systems. The dividing line between WZ Sge and SU Uma systems is moved to the dashed position if the tidal force becomes to weak to truncate the disk (see text).

that what is occurring during outbursts of WZ Sge stars is just this process.

\section{New classification of cataclysmic variables}

In Fig. 2 we show the tidal truncation radius and the $3: 1$ resonance radius as a function of the mass ratio (see also Hellier 2001) and in addition the 2:1 resonance radius. This third line in our diagram, the 2:1 resonance radius, now allows a new classification of CVs. CV systems can be divided into three groups according to their mass ratio: (1) high mass ratio systems in which the tidal truncation radius is smaller than the $3: 1$ resonance radius correspond to the U Gem-type dwarf novae, (2) intermediate mass ratio systems in which the 3:1 resonance radius is smaller than the tidal truncation radius, but the tidal truncation radius is smaller than the $2: 1$ resonance radius, correspond to the ordinary SU UMa stars, (3) lowest mass-ratio systems in which the $2: 1$ resonance radius is between the tidal truncation radius and the 3:1 resonance radius correspond to WZ Sge stars.

The exact location of the boundaries between the three cases is uncertain since the tidal truncation radius (taken here as $90 \%$ of the radius of the Roche lobe) is rather approximate. In particular, the boundary between the SU UMa stars and the WZ Sge stars in Fig. 2 is found to be around $q=0.025$, which seems to be too small as compared with $q \simeq 0.08$ obtained in Sect. 3. In those binary systems with the extreme low mass ratio, the tidal force will be weak and the ordinary tidal torques at the tidal truncation radius may not be strong enough to stop sudden expansion of the disk caused by an outburst. In such a case, the 2:1 resonance radius could be the only place 
where the disk is effectively truncated and the boundary between the SU UMa stars and the WZ Sge stars may be at $q \simeq 0.08$ rather than $q \simeq 0.025$.

Acknowledgements. We would like to thank Emmi MeyerHofmeister for helpful discussions and technical assistance. Yoji Osaki acknowledges financial support from the Japanese Ministry of Education, Culture, Sports, Science and Technology with a Grant-in Aid for Scientific Research No. 12640237.

\section{References}

Allen, C. W. 1973, Astrophysical Quantities, 3rd edition (University London, The Athlon Press)

Anderson, N 1988, ApJ, 325, 266

Cannizzo, J. K. 1993, in Accretion Disks in Compact Stellar Systems, ed. J. Wheeler (World Scientific, Singapore), 6 Hellier, C. 2001, PASP, 113, 469

Ichikawa, S., \& Osaki, Y. 1992, PASJ, 44, 15

Hirose, M., \& Osaki, Y. 1990, PASJ, 42, 135

Kato, T., Nogami, D., Baba, H., et al. 1996, PASJ, 48, L21
Leibowitz, E. M., Mendelson, H., Bruch, A., et al. 1994, ApJ, 421,771

Lin, D. N. C., \& Papaloizou, J. C. B. 1979, MNRAS, 186, 799

Lubow, S. H. 1991, ApJ, 381, 259

Matsumoto, K., Nogami, D., Kato, T., et al. 1998, PASJ, 50, 405

Meyer, F., \& Meyer-Hofmeister, E. 1981, A\&A, 104, L10

Meyer, F., \& Meyer-Hofmeister, E. 1999, A\&A, 341, L23

Osaki, Y. 1974, PASJ, 26, 429

Osaki, Y. 1989, PASJ, 41, 1005

Osaki, Y. 1995, PASJ, 47, 47

Osaki, Y. 1996, PASP, 108, 39

Osaki, Y., Meyer, F., \& Meyer-Hofmeister, E. 2001, A\&A, 370, 488

Paczynski, B. 1977, ApJ, 216, 822

Papaloizou, J. C. B., \& Pringle, J. E. 1977, MNRAS, 181, 441

Patterson, J., McGraw, J. T., Coleman, et al. 1981, ApJ, 248, 1067

Patterson, J., Augusteijn, T., Harvey, et al. 1996, PASP, 108, 748

Smak, J. 1993, Acta Astron., 43, 101

Warner, B. 1995, Cataclysmic Variable Stars (Cambridge University Press)

Whitehurst, R. 1988, MNRAS, 232, 35

Whitehurst, R. 1994, MNRAS, 266, 35 